\title{
Vitamin d deficiency impacts on expression of toll-like receptor-2 and cytokine profile: a pilot study
}

Samar Ojaimi ${ }^{1}$, Narelle A Skinner ${ }^{2,3}$, Boyd JG Strauss ${ }^{2}$, Vijaya Sundararajan ${ }^{2}$, lan Woolley ${ }^{1,2}$ and Kumar Visvanathan ${ }^{1,2,3^{*}}$

\begin{abstract}
Background: Vitamin D is believed to play an important role outside the endocrine system in the regulation of the immune system, and in cellular proliferation and differentiation. The aim of the study was to investigate the impact of vitamin D levels on innate immunity.

Methods: Participants for this prospective, longitudinal study were recruited amongst otherwise healthy staff of a large hospital in Victoria, Australia. Those fulfilling the inclusion criteria, including a vitamin D level of $<50 \mathrm{nmol} / \mathrm{L}$, were supplemented. Using flow cytometry, expression of the innate immune receptors TLR2, TLR4 and CD86 was measured on peripheral blood mononuclear cells (PBMCs) collected prior to vitamin D treatment and then at 1 and 3 months. Additonally, PBMCs at each timepoint were stimulated with specific TLR ligands and resultant supernatants were assayed for the cytokines TNFa, IL-6, IFN- $a$ and IP-10.

Results: In participants whose vitamin D level was $>100 \mathrm{nmol} / \mathrm{L}$ post supplementation ( $n=11)$, TLR2 expression on PBMCs increased significantly, with no change noted in TLR4 or CD86 expression. Stimulation of vitamin D deficient samples with TLR ligands produced a number of proinflammatory cytokines, which were significantly reduced upon vitamin D normalisation. In patients whose levels returned to a deficient level at 3 months despite ongoing low-level supplementation, an increase in the pro-inflamamtory state returned. This suggests that vitamin D may play an important role in ensuring an appropriate baseline pro-inflammatory state.
\end{abstract}

Conclusions: This ex-vivo pilot study adds clinical evidence supporting a possibly important role for vitamin D in innate immunity. If confirmed, this unique clinical study has potentially significant implications for the treatment of a variety of inflammatory conditions, where achieving optimal vitamin D levels may help reduce inflammation.

Keywords: Vitamin D, Vitamin D deficiency, Toll-like receptor, Immunity, Innate immunity

\section{Background}

Vitamin D's importance outside the endocrine system is being increasingly recognized, in particular in the regulation of hormone secretion and the immune system, as well as cellular proliferation and differentiation [1]. Consequently, vitamin D deficiency has been linked to an increased risk of a number of conditions, both infectious and non-infectious, including Mycobacterium tuberculosis, cardiovascular disease, asthma, varying

\footnotetext{
* Correspondence: kv@unimelb.edu.au

'Department of Infectious Diseases, Southern Health, Clayton Rd, Clayton,

Victoria, Australia

${ }^{2}$ Department of Medicine, Southern Clinical School, Monash University,

Clayton Rd, Clayton, Victoria, Australia

Full list of author information is available at the end of the article
}

malignancies and autoimmune conditions such as multiple sclerosis and type I diabetes [2-5].

The role of vitamin $\mathrm{D}$ with respect to adaptive immunity has been recognised for over 25 years, where it predominantly exhibits an inhibitory or suppressive effect on T-cell activation and proliferation, especially of $\mathrm{T}$ helper-1 cells (TH1), which produce IFN- $\gamma$ and lead to activation of macrophages $[1,2,6]$. However, it also favours a T helper-2 (TH2) response by the regulation of the secretion of other cytokines, resulting in increased production of IL-4, IL- 5 and IL-10 by T-cells $[1,2,7,8]$.

More recently, there is evidence that vitamin D may play an important role in innate immunity. The innate immune system involves the activation of transmembrane pattern

\section{Biomed Central}


recognition receptors such as Toll-Like Receptors (TLRs) that interact with specific pathogen proteins $[1,2]$. This in turn leads to release of antimicrobial peptides and the subsequent killing of the organism $[1,2]$. TLRs are also instrumental in activating adaptive immunity [9]. TLR2 senses lipopeptides from bacteria and leads to activation of NF- $\mathrm{KB}$ and induction of cytokine production and release $[10,11]$. TLRs also lead to expression of T-cell costimulatory molecules CD80 and CD86 via induction of IFN $\beta[11,12]$. These molecules are expressed on antigen presenting cells and are also important for B-cell activation [13]. Both TLR2 and TLR4 on monocytes have been shown to be downregulated by the immunomodulatory effect of vitamin D [14]. TLR2 has also been shown to be important in response to vaccines, with TLR2-knock out mice having reduced responses to vaccination with lipopeptides [11]. These studies prompted us to assess further the role of vitamin D on the innate immune system, specifically TLR2.

In this prospective, longitudinal pilot study, we aimed to explore whether vitamin D deficiency results in an increased innate inflammatory signature and enhanced TLR activation. No prospective data with repeated measures of vitamin D levels and markers of innate immunity are present in the published literature.

\section{Methods}

\section{Study design and subjects}

This study was designed as a pilot prospective longitudinal study in which otherwise healthy vitamin D deficient participants were followed over a period of three months after supplementation. Following approval from the institutional ethics committee, participants were recruited within the Southern Health Care Network, Victoria, Australia. Recruitment occurred at the end of Winter to maximize the chance of vitamin D deficiency.

After obtaining informed consent, participants went on to have further testing if they met the basic screening inclusion criteria, which included: being male (between 18 and 50 years of age) or female ( $>18$, with menstruation within the last year) and having a Body Mass Index (BMI) between 20 and 30. Vitamin deficiency was defined as $25-\mathrm{OH}$-vitamin D levels of $<50 \mathrm{nmol} / \mathrm{L}$. Participants were also screened for conditions that may affect vitamin D metabolism and levels including a history of renal, hepatic, thyroid, parathyroid, coeliac or other malabsorptive diseases. Those with any such conditions were excluded from the study. Those with chronic diseases, or conditions which may be associated with elevated inflammatory states, were also excluded, including diabetes, chronic infections such as chronic hepatitis and Human Immunodeficiency Virus infections, autoimmune conditions, asthma or cardiovascular diseases. Participants were also screened on history for symptoms suggestive of active infection.

A summary of the study protocol is outlined in Figure 1.

\section{Vitamin D supplementation}

Initially, all participants had baseline bloods measured. Those who were vitamin $\mathrm{D}$ deficient were then loaded with a single tablet of 50,000 units of vitamin D, given daily for 10 days. This formulation had not at the time been approved in Australia. However, it had received ethics approval in our hospital for use in the tuberculosis clinic and had been approved for routine use in other jurisdictions overseas. After loading, subjects went on to a maintenance regimen, which included one tablet $(50,000$ units) monthly. This continued for a total of 3 months of therapy, with a total of two monthly maintenance doses. After this period, with the completion of the study, participants were encouraged to continue with vitamin D supplementation at 1,000 units/day.

\section{Blood specimens}

As outlined in Figure 1, 20 mls of blood was collected in a Lithium-heparinized tube at baseline, one-month and

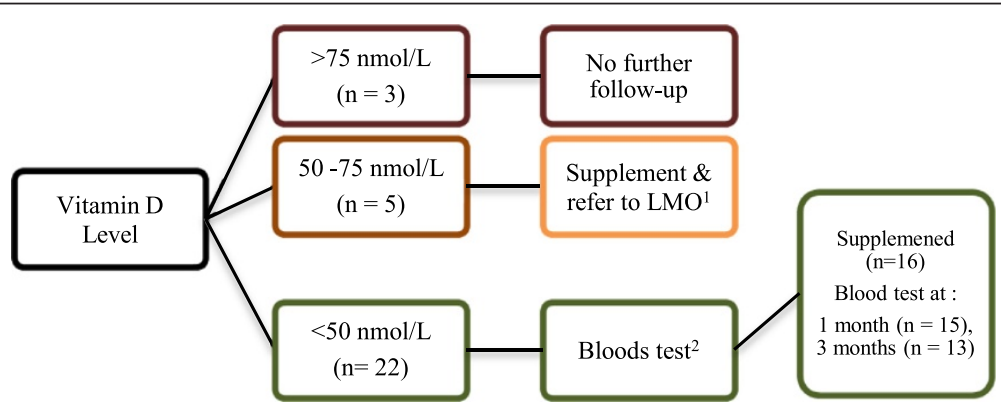

Criteria: Male $(>18,<50)$, Female $(>18$, premenopause), Body Mass Index (BMI) $<30$, no infection, autoimmunity or malabsorption. ${ }^{1}$ LMO: Local Medical Officer. ${ }^{2}$ Bloods: FBC, LFT, PTH, Ca/PO4, CRP, ESR, TSH, Coeliac screen, $20 \mathrm{mls}$ of Li-Hep blood for PBMCs

Figure 1 Summary of study protocol. 
three-month stages. Bloods analysed at one month were taken just prior to the first monthly dose, whereas those at the three-month stage were taken 1 month after the second monthly dose. Peripheral blood mononuclear cells (PBMCs) were isolated by Ficoll-gradient centrifugation and stored in liquid nitrogen for batch stimulation and flow cytometry. Plasma was also stored at $-80^{\circ} \mathrm{C}$.

Participants' vitamin D levels were measured using the Liaison 25-OH-vitamin D assay (DiaSorin SpA, Saluggia, Italy), a direct competitive chemiluminescent immunoassay.

\section{Laboratory analysis \\ Flow cytometry for TLR expression}

Two million PBMCs were stained for flow cytometry with the following anti-human monoclonal antibodies: anti-TLR2-FITC (clone TLR2.1, eBioscience, San Diego, CA), anti-TLR4-PE (clone HTA125, eBioscience), antiCD14-APC Cy7 (MФP9, BD Biosciences, San Jose, CA) and anti-CD86 (clone 2331 FUN-1, BD Biosciences). Ten thousand CD14positive monocytes were acquired on a BD FACS Canto. FlowJo software (Treestar, Ashland, OR) was used for analysis and the geometric mean fluorescence of each sample was normalized to its own isotype-matched control.

\section{Cytokine production following in vitro PBMC stimulation with TLR ligands}

PBMCs were rapidly thawed and viability determined by Trypan Blue exclusion (all samples were greater than 95\%). One million PBMCs were stimulated in polystyrene tubes in 1ml of RPMI-1640 supplemented with 5\% Foetal Calf Serum (FCS) and penicillin / streptomycin containing either 100 ng/ml Pam3Cys (tripalmitoyl-S-glycerylcysteine, Invivogen, San Diego, CA), $10 \mu \mathrm{g} / \mathrm{ml}$ PolyI:C (Invivogen), $100 \mathrm{ng} / \mathrm{ml}$ LPS (lipopolysaccharide) from Escherichia coli strain K235 (Sigma Lifesciences), 5 \%g/ml R848 (Invivogen), $0.3 \mu \mathrm{M}$ CpG 2006 or unstimulated (media alone). After 24 hours incubation at $37^{\circ} \mathrm{C}, 5 \% \mathrm{CO}_{2}, 95 \%$ humidity, the supernatants were collected and stored at $-20^{\circ} \mathrm{C}$ for cytokine ELISA.

\section{Cytokine ELISA}

The culture supernatants were assayed using ELISA, for TNF, IP-10, IL-6 (BD Biosciences, San Jose, CA) and IFN $\alpha$ (BenderMedSystems, Vienna, Austria) according to the manufacturers specifications. Assay sensitivities were $8 \mathrm{pg} / \mathrm{ml}$ for TNF, IP-10 and IFN $\alpha$ and $5 \mathrm{pg} / \mathrm{ml}$ for IL-6.

\section{Statistical methods}

We first compared TLR and cytokine levels for participants who were vitamin $\mathrm{D}$ deficient at baseline and normalised after supplementation to levels $>100 \mathrm{nmol} / \mathrm{L}$ at 1 month, $(\mathrm{n}=11)$. After this, TLR and cytokine levels were compared at 1 month and 3 months for those participants whose vitamin D at 3 months had returned to deficient levels $(n=9)$. P-values were calculated using the Wilcoxon Signed Rank Test with a one-sided alpha of 0.05 .

Statistical analysis was carried out using Graphpad Prism version 5 (Graphpad Software, La Holla, California).

\section{Results}

Out of 30 healthy staff volunteers, who were a mixture of doctors, clinical and research laboratory staff, 22 (73\%) had a vitamin D level of $<50 \mathrm{nmol} / \mathrm{L}$. Baseline characteristics are shown in Table 1. Of these 22 deficient participants, two were excluded due to abnormal renal function and four reversed their decision to participate. At the end of the three months, 3 participants were lost to follow up, mainly due to employment relocation and inconvenience. There were no reported adverse events as a result of supplementation.

Eleven of those supplemented (61.1\%) showed an excellent response with an increase in the vitamin D level to $>100 \mathrm{nmol} / \mathrm{L}$ at one month. Nine of these 11 participants had returned to vitamin D levels below 100 $\mathrm{nmol} / \mathrm{L}$ at 3 months, despite receiving ongoing vitamin D supplementation.

As seen in Figure 2, at one month, upon stimulation of PBMCs with Pam3cys there was increased expression of TLR2 in participants who reached vitamin D levels greater than $100 \mathrm{nmol} / \mathrm{L}$ upon supplementation $(\mathrm{n}=11)$ and was subsequently reversed at 3 months for those whose vitamin D had decreased to deficient levels $(n=9)$. This pattern was not seen in the participants whose peak vitamin D level reached less than $100 \mathrm{nmol} / \mathrm{L}$ (data not shown). Participant numbers in this group were small, with only 3 participants following through to testing at 3

Table 1 Participant characteristics and results

\begin{tabular}{lccc}
\hline $\begin{array}{l}\text { Baseline } \\
\text { characteristics }\end{array}$ & $\begin{array}{c}\text { Baseline } \\
(\mathbf{n}=\mathbf{1 6})\end{array}$ & $\begin{array}{c}\mathbf{1} \text { Month } \\
(\mathbf{n}=\mathbf{1 5})\end{array}$ & $\begin{array}{c}\mathbf{3} \text { Months } \\
(\mathbf{n}=\mathbf{1 3})\end{array}$ \\
\hline Mean Age (yrs) & $33.3 \pm 7.4$ & - & - \\
Sex (M:F) & $3: 13$ & - & - \\
BMI (kg/m $\left.{ }^{2}\right)$ & $23 \pm 3$ & - & - \\
Vitamin D level & $28.9 \pm 7.9$ & $106.4 \pm 27.0$ & $77.5 \pm 28.0$ \\
(nmol/L) & & $(P<0.0001)^{*}$ & $(P=0.0002) \%$ \\
Ca (mmol/L) & $2.3 \pm 0.1$ & $2.3 \pm 0.1$ & $2.3 \pm 0.1$ \\
& & $(P=0.76)^{*}$ & $(P=0.85) \%$ \\
PTH (pmol/L) & $5.2 \pm 2.6$ & $4.1 \pm 1.7$ & $4.2 \pm 2.0$ \\
& & $(P=0.07)^{*}$ & $(P=0.07) \%$ \\
CRP (mg/L) & $1.6 \pm 2.0$ & $2.6 \pm 6.7$ & $1.3 \pm 2.1$ \\
& & $(P=0.16)^{*}$ & $(P=0.11)^{\%}$ \\
\hline
\end{tabular}

PTH - Parathyroid hormone; $C a$ - Serum Calcium; CRP - C-reactive Protein. PTH, Ca \& CRP were measured using Beckman Coulter DXC800 Analyser (Brea, CA). Reference ranges: $P T H-1.7-7 \mathrm{pmol} / \mathrm{L}$, Ca: $2.20-2.60 \mathrm{mmol} / \mathrm{L}$; Vitamin D 75-250 nmol/L; CRP 0-5 mg/L. *Value at 1 month compared to baseline. ${ }^{\%}$ at 3 month compared to baseline. 


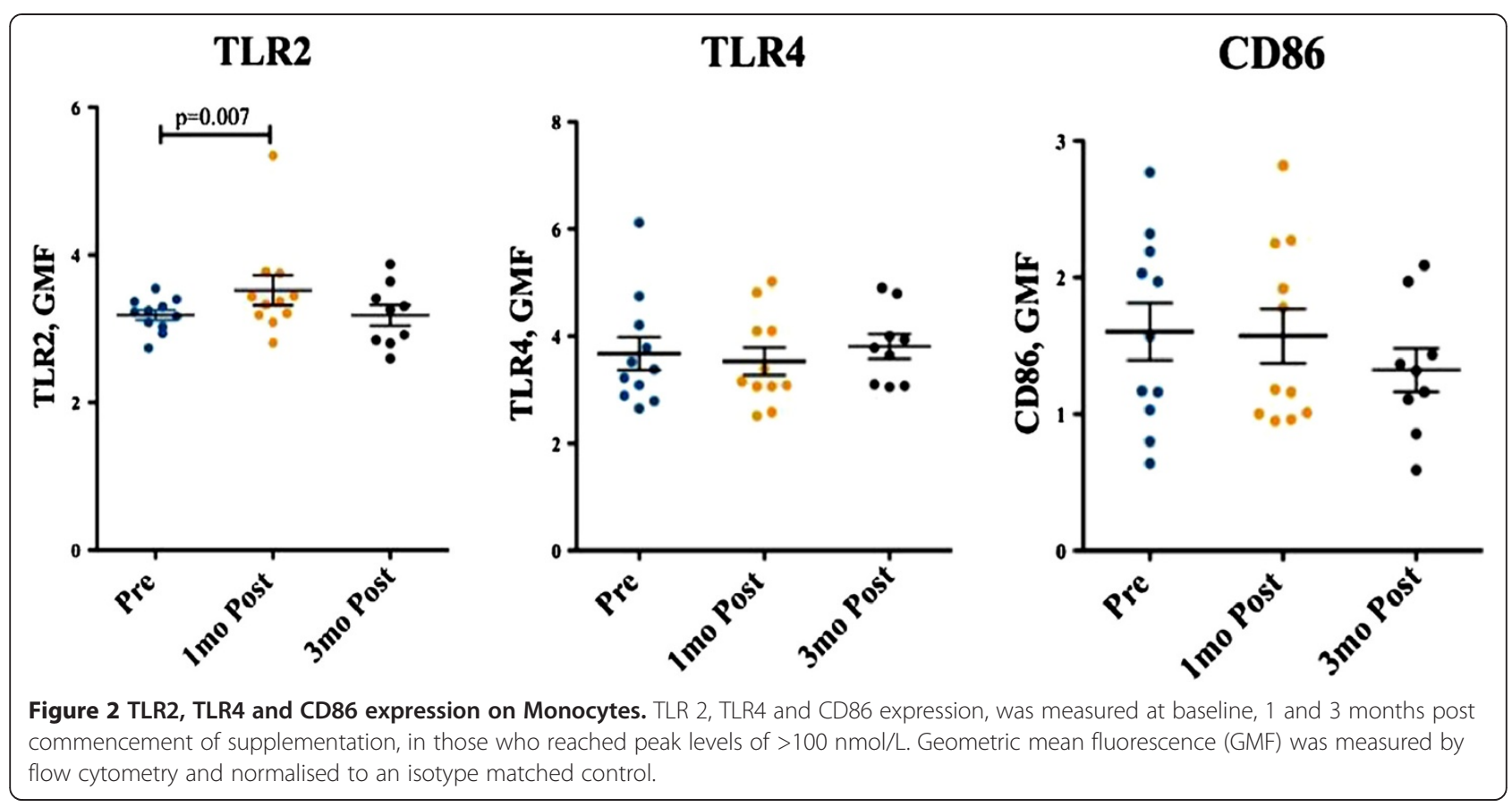

months, limiting analysis. No significant effect was seen for CD86 and TLR4 expression.

Figure $3 \mathrm{~A}$ and $3 \mathrm{~B}$ demonstrate that, in participants with levels $>100 \mathrm{nmol} / \mathrm{L}$ after one month, a statistically significant drop in TNF and IL-6 levels was seen upon TLR stimulation with LPS (TLR4 ligand) and Pam3Cys (TLR2 ligand). This indicates that, although there is no effect on TLR4 expression, vitamin D modulates the secretion of these important proinflammatory cytokines. At 3 months after the pre-load, for those with a reduction in vitamin D levels, there was a rebound rise in TNF and IL-6 levels. This drop was also seen for IP10 and IFN $\alpha$, although not statistically signsificant.

\section{Discussion}

In this small study, we have shown that supplementation of vitamin D to levels $>100 \mathrm{nmol} / \mathrm{L}$ resulted in increased expression of TLR2 on PBMCs and a reduced TLR2 stimulated cytokine profile for TNF $\alpha$, IL-6 and IFN- $\alpha$. Notably, this pattern reversed quickly when vitamin D levels reduced to $<100 \mathrm{nmol} / \mathrm{L}$ at 3 months after the initial load with vitamin D supplementation. TLR4 and CD86 expression were not affected by vitamin D deficiency in our study.

Vitamin D is obtained mostly via cutaneous production with ultraviolet $B$ radiation exposure to skin [15]. It is then further metabolised in the liver and kidney into its active form $1,25(\mathrm{OH})_{2} \mathrm{D}$ [4]. In assessing vitamin $\mathrm{D}$ status, 25(OH)D levels are mostly measured. Levels can be affected by multiple factors, including sun exposure, dietary intake, race, age and BMI $[4,16,17]$. Hence, reference ranges are controversial, without an absolute consensus on normal ranges $[4,16,18]$. It is likely that ranges will vary for individuals with similar sun exposure, possibly relating to genetic variation in enzyme activity due to polymorphisms [15]. Vitamin D levels have also been shown to be inversely related to obesity, as measured by the BMI $[17,19,20]$. Some studies of highly UV-exposed adults have shown low vitamin D levels in individuals despite similar exposure, with 'low' levels potentially being normal for these individuals [4]. However, despite variable reports of prevalence, it has become commonly accepted that levels below $30 \mathrm{ng} / \mathrm{ml}$ (75 nmol/L) are considered to be suboptimal [4]. For calcium absorption, it is believed that a level of $80 \mathrm{nmol} / \mathrm{L}$ or higher is optimal [21], whereas for fracture risk, falls, cancer, insulin sensitivity and potentially immune function, levels as high as $120 \mathrm{nmol} / \mathrm{L}$ may be necessary [21]. One study showed improved sputum conversion and radiological appearance of tuberculosis infection in those supplemented with $0.25 \mathrm{mg} /$ day (equivalent to 10,000 IU of per day) [22]. In a similar study, Martineau et al. showed the same effect only in participants with the $t t$ genotype of the TaqI vitamin D receptor polymorphism, with the mean 25-hydroxyvitamin $\mathrm{D}$ level being just over $100 \mathrm{nmol} / \mathrm{L}$ [23]. In another study, respiratory infections were reduced in African American women who were receiving 2000 IU per day with serum levels up to $87 \mathrm{nmol} / \mathrm{L}$ [21]. Most experts agree, based on prior provocative testing and other studies, that vitamin $D$ deficiency is at levels of $<20 \mathrm{ng} / \mathrm{mL}$ $(50 \mathrm{nmol} / \mathrm{L})$; insufficiency, $21-29 \mathrm{ng} / \mathrm{ml}(50$ to $75 \mathrm{nmol} / \mathrm{L}$ ); 

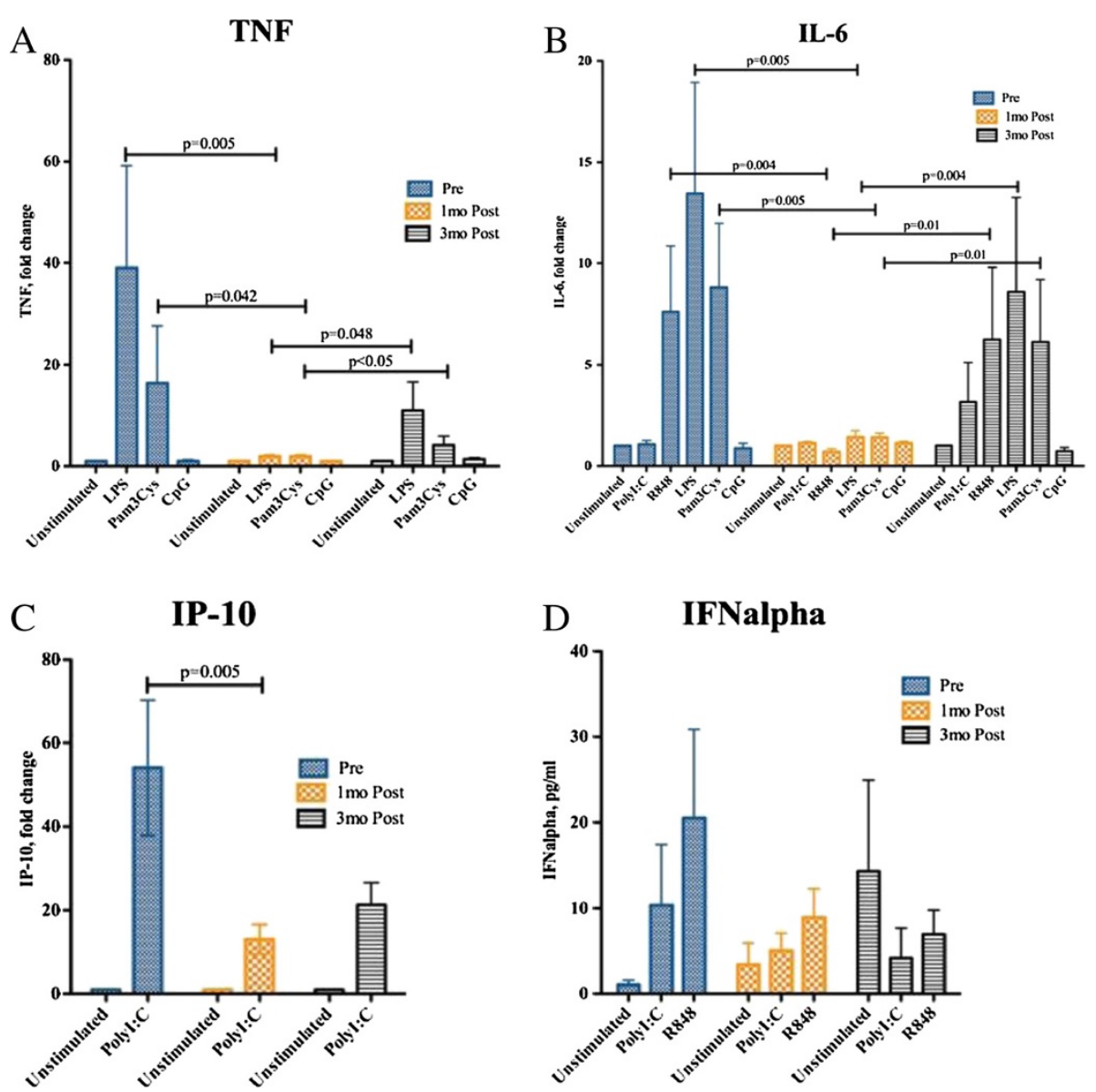

Figure 3 Cytokine concentrations upon stimulation with TLR specific ligands, as measured at baseline, 1 and 3 months post start of vitamin D supplementation. PBMCs were stimulated with TLR ligands LPS (TLR4), Pam3Cys (TLR2), CPG (TLR9) for 24 hours and supernatants were assayed by cytokine ELISA for TNF (Figure 3A) and IL-6 (Figure 3B). Results were expressed as fold change compared to unstimulated PBMCs. In Figures 3C and 3D, PBMCs were stimulated with TLR ligands Polyl:C (TLR3) and R848 (TLR7/8) and supernatants were assayed by ELISA for IP-10 (Figure 3C) and IFNalpha (Figure 3D). Results were expressed by fold change for Figure 3C and absolute values for Figure 3D.

the recommended level $>32 \mathrm{ng} / \mathrm{mL}(80 \mathrm{nmol} / \mathrm{L})$; with an upper limit of $100 \mathrm{ng} / \mathrm{mL}(250 \mathrm{nmol} / \mathrm{L})$ [18,24]. Our pilot study supports levels $>100 \mathrm{nmol} / \mathrm{L}$ to maintain adequate innate immune effects, as statistically significant reductions of proinflammatory cytokines and increased TLR expression upon stimulation were seen only in study participant who reached such levels. The increased TLR expression and the reciprocal reduction in the proinflammatory cytokine response were lost upon reduction of vitamin $\mathrm{D}$ levels back to below $100 \mathrm{nmol} / \mathrm{L}$.

Mononuclear cells and various other cells in the body, including prostate, lung and colon cancer cells, contain $1 \alpha(\mathrm{OH})$ ase, the enzymatic machinery required to produce active vitamin $\mathrm{D}$, which in turn works by binding to the vitamin $\mathrm{D}$ receptor (VDR), a specific nuclear receptor [8]. VDR is a ligand dependent transcription factor belonging to the superfamily of steroid, thyroid hormone and retinoid nuclear receptors [8]. Upon binding to VDR, the active form of vitamin $\mathrm{D}$ results in the production of cathelicidin, an antimicrobial peptide involved in the killing of mycobacteria [1]. Prior studies have shown increased TLR activation after stimulation of $1 \alpha(\mathrm{OH})$ ase and (VDR) [5,25,26]. Walker et al. showed that monocytes, derived from cord blood, exhibited decreased TLR-induced cathelicidin expression when cultured in vitamin D deficient plasma [27]. Liu et al. had previously also shown that TLR activation up-regulated VDR expression and subsequent induction of cathelicidin [26]. Lack of vitamin D, VDR or $1 \alpha(\mathrm{OH})$ ase blunts the macrophages ability to produce antimicrobial peptides [2].

While multiple studies have looked at the role of TLR2 and TLR4 activation on the expression of VDR and cathelicidin, few are available on the effect of vitamin D on TLR activation. Do et al. showed, in a cross sectional study of patients with Behçet's disease, an inverse correlation between the expression of TLR2 and TLR4 and vitamin D levels. Additonally, in vitro, they demonstrated that vitamin D3 was able to suppress 
protein and mRNA expression of both TLR2 and TLR4 [9]. In contrast, our study showed an increase in TLR2 expression upon supplementation of vitamin $\mathrm{D}$ to levels above $100 \mathrm{ng} / \mathrm{mL}(250 \mathrm{nmol} / \mathrm{L})$. This fits with the hypotheses that vitamin $\mathrm{D}$ is an immune modulator which varies in its effect based on the presence of inflammation. In the setting of Behçet's, an inflammatory condition, vitamin D may act as a down regulator of inflammatory responses, resulting in reduced TLR expression. In healthy subjects, vitamin D may heighten TLR expression, in preparation for possible pathogen encounters. These hypotheses need to be investigated further, ideally in a large study which includes both healthy subjects and patients with other conditions, including chronic inflammatory conditions and chronic infections. There is recent evidence that vitamin D's immunomodulatery effect is in part due to the presence of VDR on inflammatory cells and the ability of macrophages and dendritic cells to produce the active form of vitamin D in the presence of CYP27B1 (1 $\alpha$ hydroxylase) [28]. In these cells however, the enzyme is stimulated by cytokines rather than parathyroid hormone, which stimulates this enzyme in the kidney [28]. Stimulation of TLR2 has been shown to result in increased expression of CYP27B1 [25].

Mahon et al. previously investigated the association between vitamin $\mathrm{D}$ and cytokine production while examining the effect of vitamin D supplementation in multiple sclerosis patients [26]. They found that supplementation significantly increased transforming growth factor (TFG)$\beta 1$, an anti-inflammatory cytokine, without a significant difference in TNF- $\alpha$ and IFN $\gamma$ levels [29]. However, vitamin D levels in their subjects were below recommended levels despite supplementation [29], limiting interpretation of their results.

There are a number of limitations to our study. As a pilot study, the sample size was small. However, with repeated measures of both vitmain $\mathrm{D}$ levels and innate immune markers, we were able to efficiently use our data to demonstrate reversible changes in innate immunity when vitamin D levels changed from deficient to normal. In order to be able to properly assess the impact of different vitamin D serum levels and to explore further whether supplementation of the vitamin to levels $>100$ $\mathrm{nmol} / \mathrm{L}$ is optimal, a larger study, with a similar repeated measures design which lends itself to investigating the reversability of vitamin D's effect on TLR expression and cytokine production, is required. It would be important to objectively assess adherence to supplementation, such as pill-count or direct observed therapy protocols. Our study relied on word-of-mouth and may have thus been influenced by non-adherence. It would also be worthwhile to include patients with chronic inflammatory conditions and infections, in order to assess if vitamin D supplementation in these patients affects their cytokine profile. Another relevant question not addressed by this study is the clinical and functional significance of these findings. A larger study with longer supplementation and follow-up periods, allowing monitoring for rates infection or inflammatory conditions, may have permitted analysis of the potential clinical relevance of the demonstrated increase in expression of TLR2. This would have been made more relevant by comparison to an unsupplemented control group with variable vitamin D levels. In this study, an attempt was made to look at the functional meaning of the increased expression of TLR2 activation by looking at cytokine production. It would also be of interest to expand the study further in order to explore the molecular mechanisms behind our findings.

\section{Conclusions}

While this is a small, pilot study, we have shown for the first time in humans that optimal vitamin D levels after supplementation may improve the expression of TLR2 and hence the body's ability to fight infections. We have also demonstrated a marked reduction in the induced cytokine profile, specifically IL6, TNF and IFN alpha associated with higher vitamin D levels and the reversibility of the innate immune profile with decreasing levels. There are multiple implications for this, including the potential role for vitamin $\mathrm{D}$ to dampen down the innate immune system in autoimmune conditions. In addition, there may be a role for vitamin $\mathrm{D}$ as a modulator of immune response to vaccines. To help further delineate the benefits of vitamin $\mathrm{D}$ and its role in vivo, larger, randomised, double-blinded, controlled studies with broader sampling of subjects, including those with inflammatory conditions, are required.

\section{Abbreviations}

VDR: Vitamin D receptor; TLR: Toll-Like Receptors; Vitamin D: In this manuscript, mainly used for the measured form (inactive form) 25-hydroxyvitaminD [25(OH)D]); TNF: Tissue Necrosis Factor; IL: Interleukin; PBMCs: Peripheral blood mononuclear cells; IFN: Interferon; IP: Interferon gamma-induced protein; BMl: Body Mass Index.

\section{Competing interests}

The authors declare that they have no competing interests.

\section{Authors' contributions}

SO contributed to the design and coordination of the study, including the ethics submission, participant recruitement, data collection and

interpretation, as well as drafting of the manuscript. NAS performed the laboratory analysis, as well as described the methods used in doing so. BJGS contributed to the design of the study. VS performed the statistical analysis. IW contributed to the design and coordination of the study. KV was general supervisor of the study, with contribution to the conception and design of the study, as well as data analysis. All authors contributed to the critical editing and revision of the manuscript. They have all read and approved the final manuscript.

\section{Acknowledgements}

We would like to thank all volunteers who agreed to participate in this study. 


\section{Author details}

${ }^{1}$ Department of Infectious Diseases, Southern Health, Clayton Rd, Clayton, Victoria, Australia. '2Department of Medicine, Southern Clinical School, Monash University, Clayton Rd, Clayton, Victoria, Australia. ${ }^{3}$ Departments of Infectious Diseases and Medicine, Clinical Immunology Laboratory, St Vincent's Hospital, University of Melbourne, 4th Floor, Clinical Sciences Building, Fitzroy, Victoria 3065, Australia.

Received: 12 March 2013 Accepted: 1 July 2013

Published: 22 July 2013

\section{References}

1. Bickle D: Nonclassic Actions of Vitamin D. J Clin Endocrinol Metab 2009, 94:26-34.

2. Holick MF: Vitamin D: Extraskeletal Health. Endocrinol Metab Clin N Am 2010, 39:381-400

3. Souberbielle JC, Body JJ, Lappe JM, Plebani M, Shoenfeld Y, Wang TJ, et al: Vitamin D and musculoskeletal health, cardiovascular disease, autoimmunity and cancer: Recommendations for clinical practice. Autoimmun Rev 2010, 9:709-715.

4. Binkley N, Ramamurthy R, Krueger D: Low Vitamin D Status: Definition, Prevalence, Consequences, and Correction. Endocrinol Metab Clin N Am 2010, 39:287-301.

5. Maalouf NM: The noncalciotropic actions of vitamin D: recent clinical developments. Curr Opin Nephrol Hyperten 2008, 17:408-415.

6. Adams JS, Liu PT, Chun R, Modlin RL, Hewison M: VitaminD in Defense of the Human Immune Response. Ann N Y Acad Sci 2007, 1117:94-105.

7. Litonjua AA, Weiss ST: Is vitamin D deficiency to blame for the asthma epidemic? J Allergy Clin Immunol 2007, 120:1031-1035.

8. Reichrath J: Vitamin D, and the skin: an ancient friend, revisited. Exp Dermatol 2007, 16:618-625.

9. Do JE, Kwon SY, Park S, Lee ES: Effects pf vitmain D on expression of Toll-like receptors of monocytes from patients with Behçet's disease. Rheumatology 2008, 47:840

10. Bekeredjian-Ding I, Jego G: Toll-like receptors - sentries in the B-cell response. Immunology 2009, 128:311-323.

11. McCormack WJ, Parker AE, O'Neill LA: Toll-like receptors and NOD-like receptors in Rheumatic Diseases. Arthritis Res \& Therapy 2009, 11:243-251.

12. Muzio M, Mantovani A: Toll-like receptors. Microbes and Infection 2000, 2:251-255.

13. Rau FC, Dieter J, Luo Z, Priest SO, Baumgarth N: B7-1/2 (CD80/CD86) Direct Signalling to B Cells Enhances IgG Secretion. J Immunol 2009, 183:7661-7671.

14. Sadeghi $K$, Wessner $R$, Laggner U, Ploder M, Tamandl $D$, Josef F, et al: Vitamin D3 down-regulates monocyte TLR expression and triggers hyporesponsiveness to pathogen-associated molecular pattern. Eur J Immunol 2006, 36:361-379.

15. Christakos S, Ajibade DV, Dhawan P, Fechner AJ, Mady LJ: Vitamin D: Metabolism. Enodcrinol Metab Clin N Am 2010, 39:243-253.

16. Mosekilde L: Vitamin $D$, requirement and setting recommendation levels: long-term perspectives. Nutr Rev 2008, 66:S170-S177.

17. Muscogiuri G, Sorice GP, Prioletta A, Policola C, Della Casa S, Pontecorvi A, et al: 25-Hydroxyvitamin D Concentration Correlates with InsulinSensitivity and BMI in obesity. Obesity 2010, 18:1906-1910.

18. Holick MF, Vitamin D: Status: Measurement, Interpretation, and Clinical Application. Ann Epidemiol 2008, 19:73-78.

19. Vashi PG, Lammersfeld CA, Braun DP, Gupta D: Serum 25-hydroxyvitamin D is inversely associated with body mass index in cancer. Nutr J 2011, 10:51-57.

20. Konradsen S, Ag H, Lindberg F, Hexeberg S, Jorde R: Serum 1,25-dihydroxy vitamin D is inversely associated with body mass index. Eur J Nutr 2008, 47:87-91.

21. Heaney RP: Vitamin D: criteria for safety and efficacy. Nutr Rev 2008, 66:S178-S181.

22. Nursyam EW, Amin Z, Rumende CM: The Effect of Vitamin D as Supplementary Treatment in Patients wiht Modertely Advanced Pulmonary Tuberculous lesion. Acta Med Indones 2006, 38:3-5.

23. Martineau AR, Timms PM, Bothamley GH, Hanifa Y, Islam K, Claxton AP, et al: High-dose vitamin D3 during intensive-phase antimicrobial treatment of pulmonary tuberculosis: a double-blind randomised controlled trial. Lancet 2011, 377:242-250.
24. Hollis BW: Assessment and Interpretation of Circulating 25Hydroxyvitamin D and 1,25-Dihydroxyvitamin D in the clinical environment. Endocrinol Metab Clin N Am 2010, 39:271-286.

25. Adams JS, Ren S, Liu PT, Chun RF, Lagishetty V, Gombart AF, et al: Vitamin D-Directed Rheostatic Regulation of Monocyte Antibacterial Responses. J Immunol 2009, 182:4289-4295.

26. Liu PT, Stenger S, Li H, Wenzel L, Tan BH, Krutzik SR, et al: Toll-like Receptor Triggering of a Vitamin D-Mediated Human Antimicrobial Response. Science 2006, 311:1770-1773.

27. Walker VP, Zhang X, Rastegar I, Liu P, Hollis BW, Adams JS, et al: Cord Blood Vitamin D Status Impacts Innate Immity Responses. J Endocrinol Metab 2011, 96:1835-1843.

28. Bikle DD: Vitamin D, and the immune system: role in protection against bacterial infection. Curr Opin Neprhol Hypertens 2008, 17:348-352.

29. Mahon BD, Gordon SA, Cruz J, Cosman F, Cantorna MT: Cytokine profile in patients with Multiple Sclerosis following vitamin D supplementation. J Neuroimmunology 2003, 134:128-132.

doi:10.1186/1479-5876-11-176

Cite this article as: Ojaimi et al:: Vitamin d deficiency impacts on expression of toll-like receptor-2 and cytokine profile: a pilot study. Journal of Translational Medicine 2013 11:176.

\section{Submit your next manuscript to BioMed Central and take full advantage of:}

- Convenient online submission

- Thorough peer review

- No space constraints or color figure charges

- Immediate publication on acceptance

- Inclusion in PubMed, CAS, Scopus and Google Scholar

- Research which is freely available for redistribution 